\title{
Optimized Trip Angle in Accelerometer to Improve Force and Voltage Measurements and Reduce Calibration Error
}

This paper was downloaded from TechRxiv (https://www.techrxiv.org).

\section{LICENSE}

CC BY 4.0

SUBMISSION DATE / POSTED DATE

$11-02-2022$ / 19-02-2022

\section{CITATION}

Ubosi, Dozie (2022): Optimized Trip Angle in Accelerometer to Improve Force and Voltage Measurements and Reduce Calibration Error. TechRxiv. Preprint. https://doi.org/10.36227/techrxiv.19158449.v1

$\mathrm{DOI}$

10.36227/techrxiv.19158449.v1 


\title{
Optimized Trip Angle for Accelerometer for Improved Force and Voltage Measurements and Reduced Calibration Error
}

By Dozie Ubosi

Mechanical Engineering Undergraduate at the University of Guelph.

\begin{abstract}
Micro- electromechanical systems are small-scale machines that have built in electric and machine-like components. They are miniature in size and despite their size they are composed of parts made of micro actuators, micro sensors, microprocessors, components for data processing and parts that can interact with external hardware. Some Micro-electromechanical system devices convert a mechanical signal to an electrical or optical signal and can be referred to as transducers. Micro-electromechanical systems serve important purposes in commercial and enterprise technology. Some applications of Micro-electrical systems include blood pressure sensors and insulin monitors. The most revolutionary Micro-electromechanical system is the accelerometer used in airbag sensors, the technology works by a central mass moving in response to a vehicles acceleration. This mass is mounted on a hinge that limits its movements and it returns it to a central position when it rests, electrical sensors in the chip read the mass's movement and relay the data to a connected microprocessor. When the change in position reaches an unsafe level, the airbags are deployed.
\end{abstract}

\section{Introduction}

Accelerometers can detect position and acceleration by reading signals from systems. The sensor gathers information from a given system, integrated circuitry handles the logical functions whereas the Micro-electromechanical system complements the logic with control functions. Miniature accelerometers are used in phones to rotate display, game controller joysticks, robotic movements, and alarm systems. These accelerometers work by having an inertial mass, usually a rolling sphere, in them with a conductive terminal at the bottom. A good analogy relating back to computer processing is the binary coding language, if the conductive path is open the signal returned is 0 , if the conductive path is closed the signal returns 1 . When the device is inclined. This paper examines the applications of Microelectromechanical Systems accelerometers to mechatronic hands for trans radial amputees.

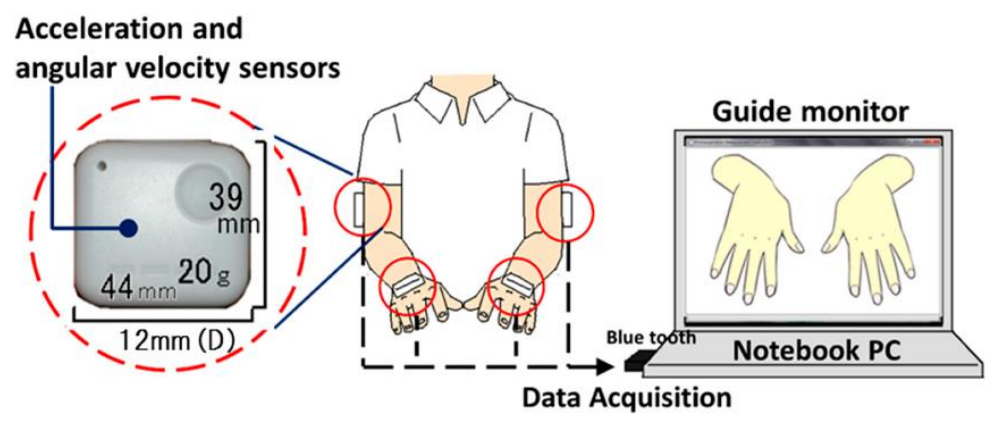

Fig 1 Example of sensors being used to measure angular velocity and acceleration of the human 

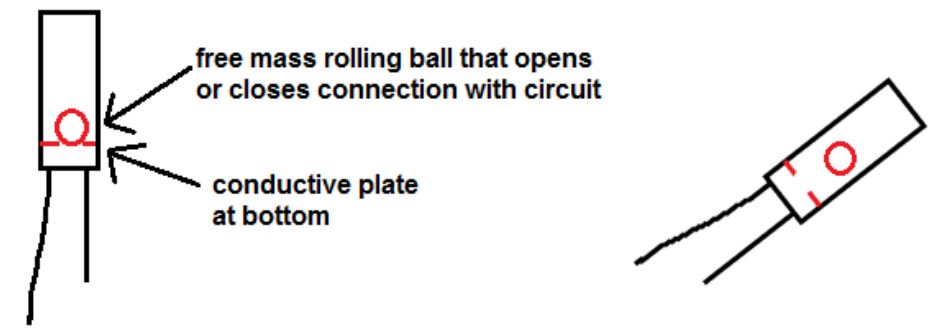

Closed circuit

Open circuit

Fig 2 Accelerometer Mechanism

\section{Device Concept and Digital Sensing}

The accelerometer package is oriented with respect to an origin specific to the given device. Equations for $x, y$, and $z$ directions can be formed

$\boldsymbol{\alpha}_{a}=f_{a}(\vartheta, 6, \zeta, \ddot{x}, \ddot{y}, \ddot{z})$

$\boldsymbol{\alpha}_{b}=f_{b}(\vartheta, 6, \zeta, \ddot{x}, \ddot{y}, \ddot{z})$

$\boldsymbol{\alpha}_{c}=f_{c}(\vartheta, 6, \zeta, \ddot{x}, \ddot{y}, \ddot{z})$

where $\theta, \beta$, and $\zeta$ are the angles of the three-axis accelerometer with respect to the $x-z, x-y$, and $z-y$ planes. The output signals are $\boldsymbol{\alpha}_{\mathrm{a}}, \boldsymbol{\alpha}_{\mathrm{b}}$ and $\boldsymbol{\alpha}_{\mathrm{c}}$. To extract the three positions requires approximations of the three angles. It can be seen from the intricacy of these system equations that the approximation of a slip signal in practice is not simple and can have associated errors. An artificial hand using input from a set of Micro-Electromechanical Systems hardware could approximate its position and tilt and would be useful in the operation of a whole arm where there are elbow and shoulder joint.

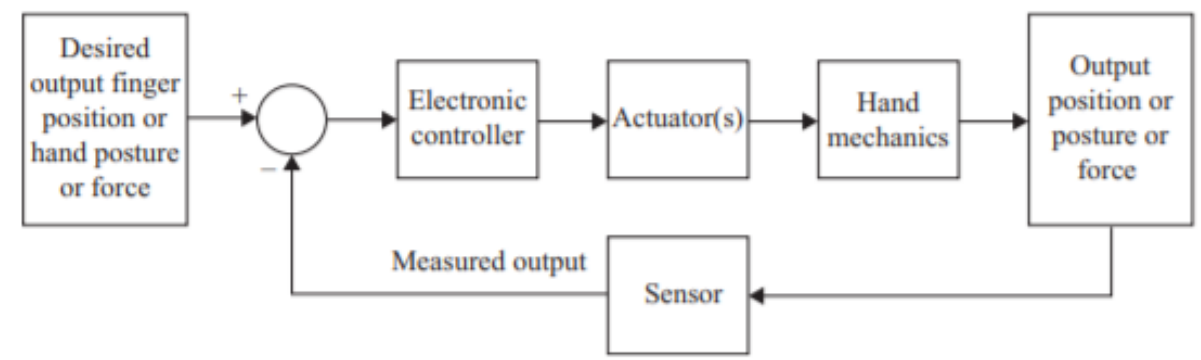

Fig 3 Block diagram of a mechatronic hand system

Accelerometers work by spotting changes in angle from an origin unique to each device. If the angular position is larger than these trip angle values from the calibration by the manufacturers in the $x, y$ or $z$ direction, a trigger will be engaged and some kind of alarm will indicate unsafe or non-working conditions. This could be applicable to detecting the force a mechatronic hand is applying to an object 
by correlating the angles in the $x, y$, and $z$ direction.

(a)

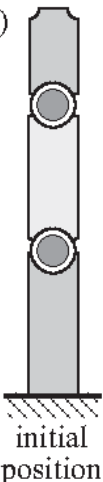

(b)

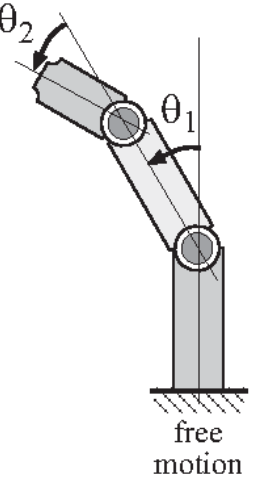

(c)

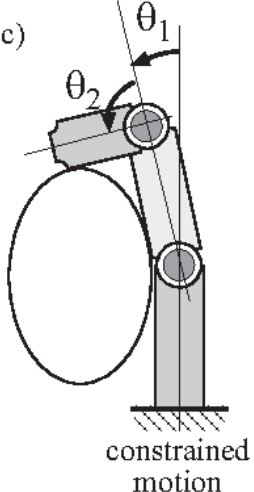

Fig 4 Angular motion of a mechatronic finger

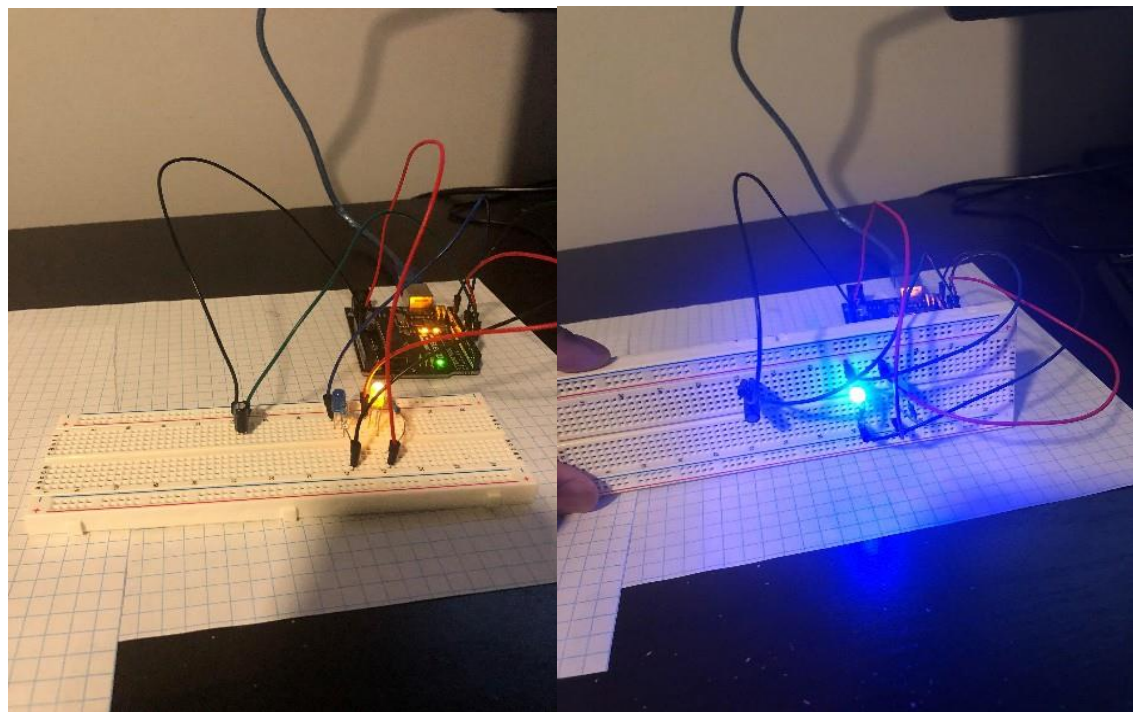

Fig 5 Experimental demonstration of an accelerometer mechanism. Yellow light indicates position is at zero plane. Blue light indicates an angular rotation of some sort.

\section{Signal Analysis and Measuring Trip Angle}

The Accelerometer is paired with a digital filter to return a binary signal 1 or 0 , indicating if the mechanism is rotated past the trip angle. 


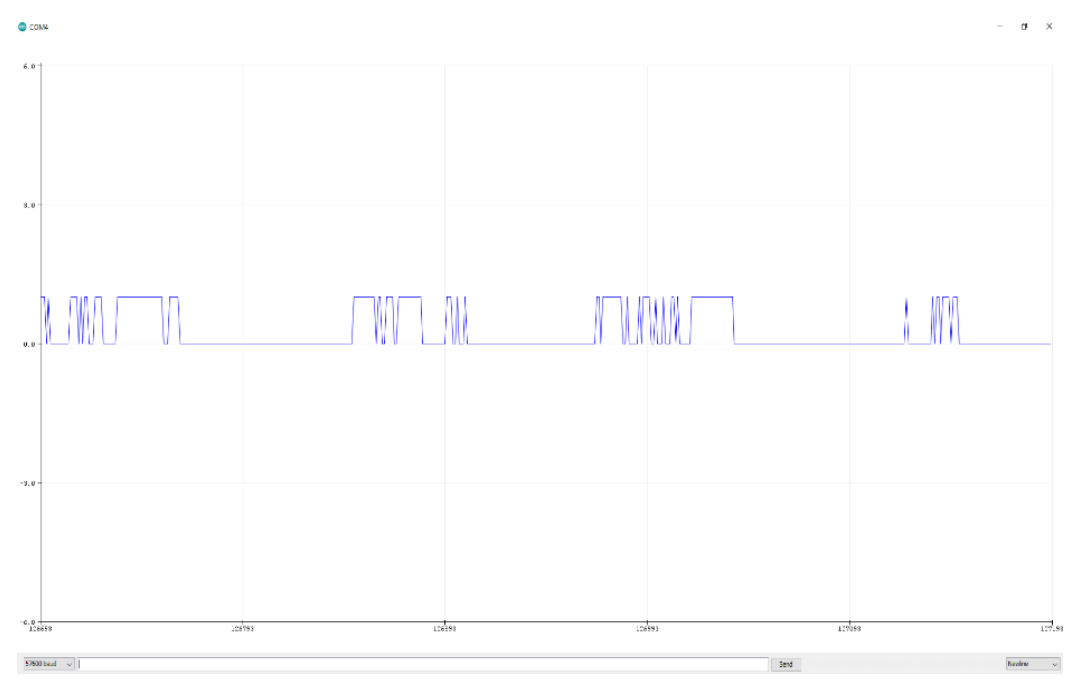

Fig 6 Complementary digital signal showing 1 when trip angle is exceeded and 0 when at zero position

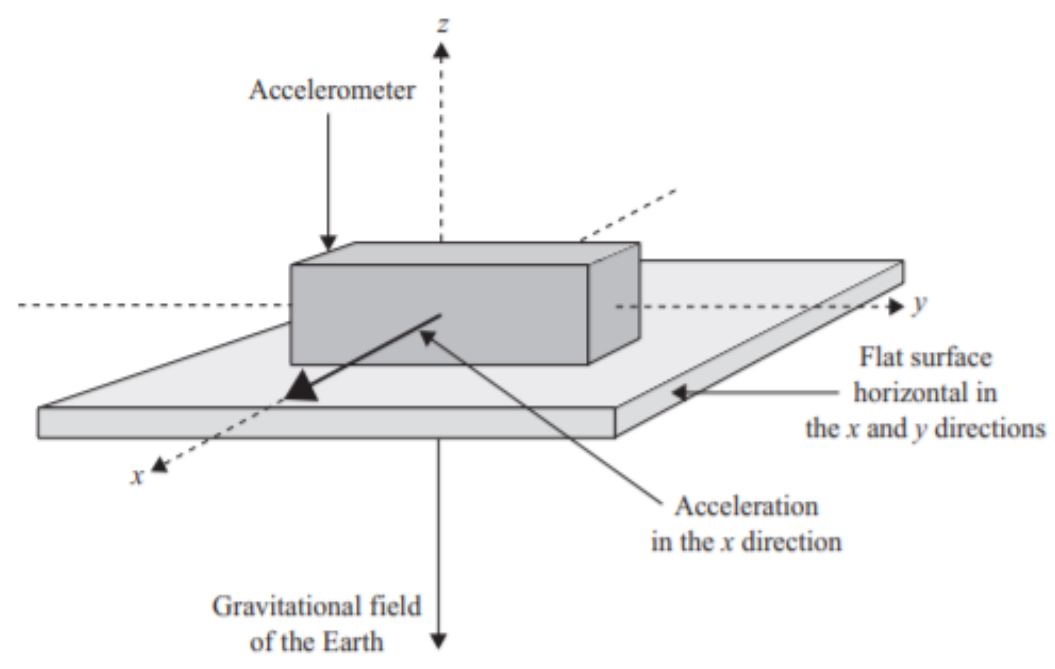

Fig 7 Accelerometer at zero reference frame

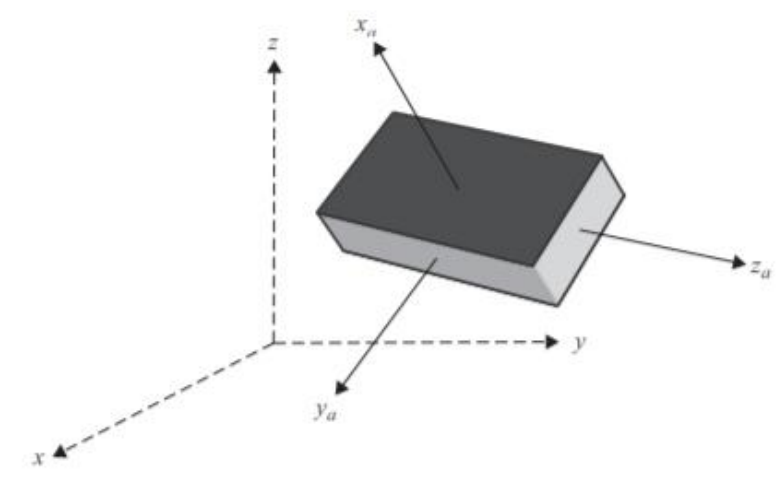

Fig 8 Accelerometer rotated to an arbitrary angle $x_{a}, y_{a}$, and $z_{a}$ with respect to zero reference frame. 

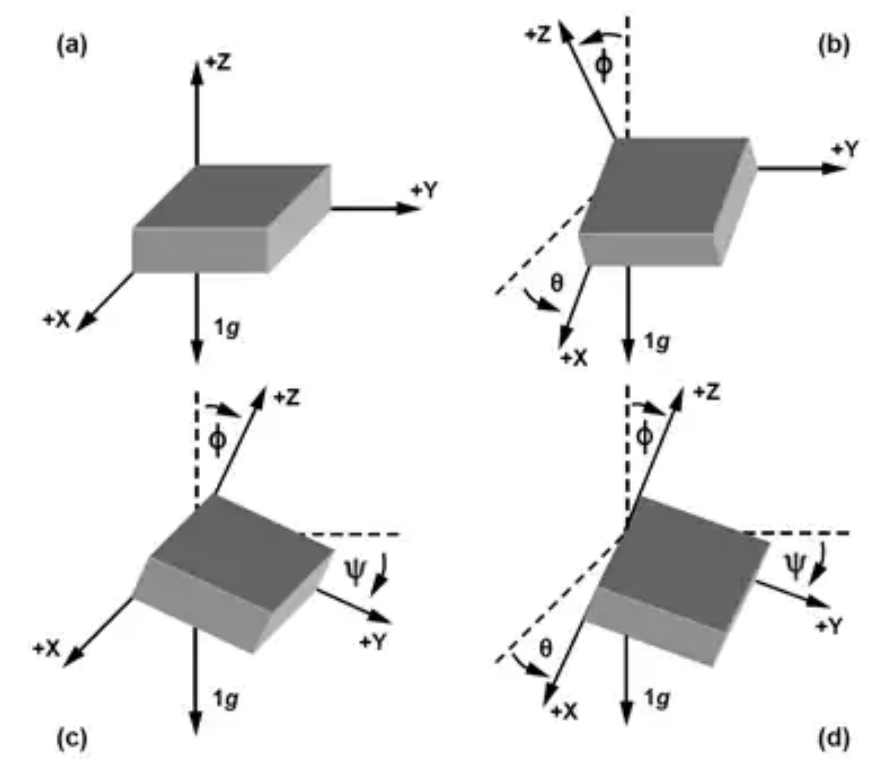

Fig 9 Accelerometer rotated at various angles

To calculate the angles of the accelerometer, simple trigonometry can be used.

$$
\begin{gathered}
\theta=\tan ^{-1}\left(\frac{A_{z}}{\sqrt{A_{y}^{2}+A_{z}^{2}}}\right) \\
\varphi=\tan ^{-1}\left(\frac{A_{y}}{\sqrt{A_{x}^{2}+A_{z}^{2}}}\right) \\
\emptyset=\tan ^{-1}\left(\frac{\sqrt{A_{x}^{2}+A_{y}^{2}}}{A_{z}}\right)
\end{gathered}
$$

Any real accelerometer is going to have some nonlinearity and offset error. As trip angle range decreases, accuracy increases therefore the trip angle must be optimized for least offset error. This is imperative as the sources of error is proportional to the number of axes the device is getting input from. 


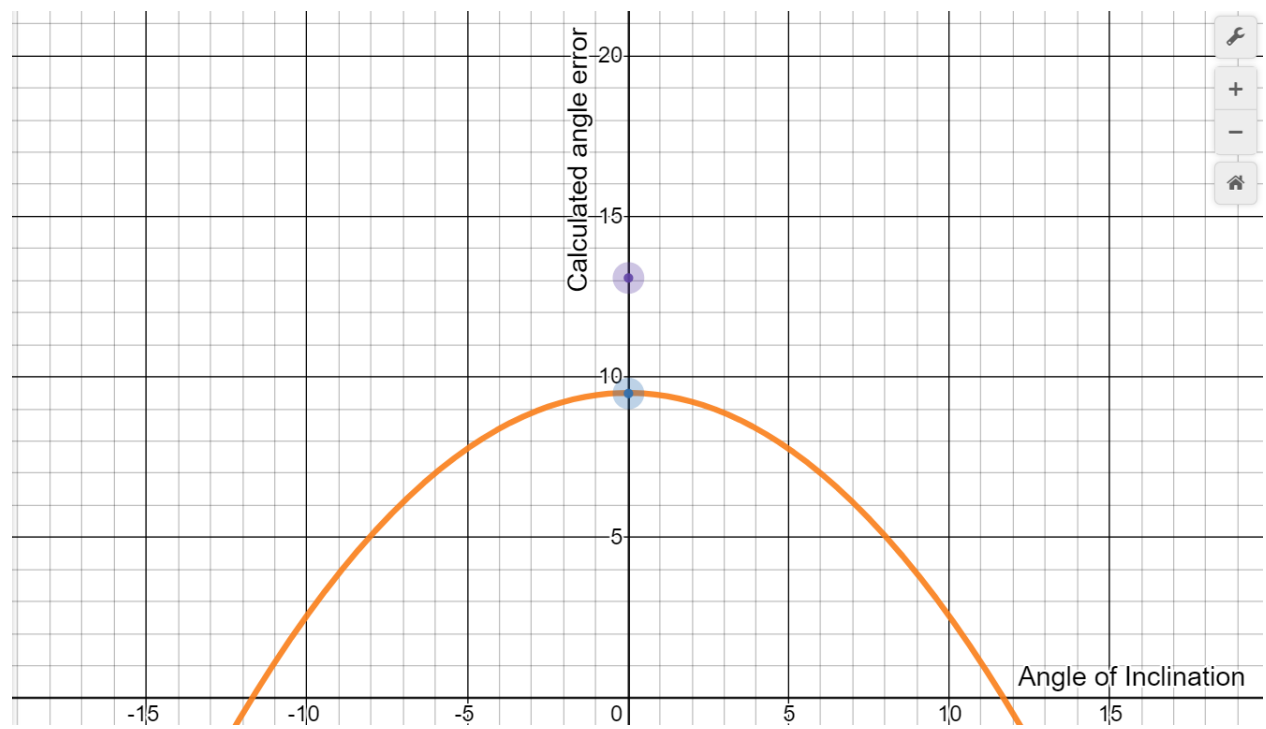

Fig 10 Offset error for an accelerometer. The $\mathrm{x}$ axis is the angle of inclination. The $y$ axis is the calculated error.

\section{Conclusion}

Micro-Electromechanical Systems have a myriad of commercial applications, one of these being the accelerometer. An accelerometer can be used to detect the orientation of a given object in a mechanism with simple trigonometry and digital filtering of signals. Trip angle range must be kept at a minimum to reduce the calculated offset error in the system, especially in a three-dimensional application. For trans radial amputees, this would benefit the devices needed to replicate human-like movements in the hands. There are a range of factors one must consider when selecting an accelerometer, things like filter frequency, switch tolerancing and number of axes. This paper dealt with triple axes accelerometer. The output signals are a function of the three angles in each given axes and the normal and tangential component of the acceleration in each direction. With this application implemented there is a vast amount of more applications in robotics design and bionic studies that can be improved by Micro-electromechanical Systems. 


\section{$\underline{\text { References }}$}

1. Zhao, \& Yeatman, E. M. (n.d.). Micro Capacitive Tilt Sensor for Human Body Movement Detection. In 4th International Workshop on Wearable and Implantable Body Sensor Networks (BSN 2007) (pp. 195-200). Springer Berlin Heidelberg.

2. Chappell. (2016). Mechatronic Hands: Prosthetic and robotic design: Prosthetic and robotic design. The Institution of Engineering and Technology.

3. Fisher, C. J. (2011, May 6). Using an accelerometer for inclination sensing. DigiKey. Retrieved February 5, 2022

4. An introduction to MEMS (micro-electromechanical systems). (n.d.). Retrieved February 5, 2022

5. Tilt switches information. Tilt Switches Selection Guide: Types, Features, Applications I Engineering360. (n.d.). Retrieved February 5, 2022 